\title{
Vascular effects of rapid-acting insulin analogs in the diabetic patient: a review
}

\author{
Steve Fordan \\ Philip Raskin \\ Department of Medicine, Division \\ of Endocrinology, University of Texas, \\ Southwestern Medical Center, Dallas, \\ TX, USA
}

\begin{abstract}
The insulin analogs lispro, aspart, and glulisine are the only commercially available rapid-acting insulins to treat diabetes. We review the evidence for treating hyperglycemia, using insulin, and specifically using rapid-acting analogs in diabetic individuals, on the prevention of vascular events. We review the beneficial effects of insulin on the vascular system, which include vasodilation and anti-inflammatory actions. The effects of treating hyperglycemia and intensive blood glucose control on vascular outcomes are reviewed.
\end{abstract}

Keywords: lispro, aspart, glulisine, nitric oxide synthetase, nuclear factor kappa B

\section{Introduction}

The epidemic of diabetes is worsening. The Centers for Disease Control estimates that over 23 million Americans suffer from diabetes, with an incidence of over 1.4 million new cases of diabetes every year. ${ }^{1}$ As the prevalence of diabetes increases, there is greater concern for the vascular complications that accompany diabetes. Insulin remains the most potent medication available to treat diabetes, and arguably to prevent diabetic complications. Some advocate using insulin much sooner in the course of diabetes. ${ }^{2}$ On the other hand, others believe that insulin may actually be harmful in obese type 2 diabetic subjects because it increases body fat, which may exacerbate insulin resistance. ${ }^{3,4}$ This review explores the interplay between insulin and the vascular system with special emphasis on the rapid-acting insulin analogs. Because of the heterogeneity of the literature, this paper will refer to vascular events defined very loosely, including peripheral events, cardiovascular events, cerebrovascular events, and death due to vascular causes.

\section{Is insulin harmful?}

The relationship between insulin and vascular events is debatable. One study has associated higher serum insulin levels as an independent predictor of cardiovascular events. The Helsinki Policeman study was a retrospective study of 22-year mortality data of 970 non-diabetic men without coronary artery disease. The authors found an increase in cardiovascular mortality among subjects with higher serum insulin levels. ${ }^{5}$ A larger study, the Paris Protection Study, followed 7246 non-diabetic men without coronary artery disease for an average of 63 months and concluded that higher fasting plasma insulin levels were an independent risk factor for the development of coronary artery disease. ${ }^{6}$

Although these studies may suggest that elevated circulating insulin is a direct cause of vascular disease, this does not seem to be true. Subjects with insulin producing neoplasms do not have an increase in clinically overt atherosclerotic disease. ${ }^{7}$ This suggests that factors other than hyperinsulinemia are responsible for an increased risk of vascular disease. Insulin resistance is often associated with hypertension, lipid abnormalities, and obesity, all of which are thought to contribute much more than 
hyperinsulinemia to the development of vascular disease. ${ }^{8}$ In fact, some have proposed that insulin resistance is another symptom associated with the metabolic syndrome and cardiovascular disease, rather than the root cause of cardiovascular disease. Cardiologists performing a population study on 322 healthy adults have described an "insulin gradient." These researchers noted a direct correlation between body weight and blood pressure and insulin levels. The heavier their subjects were, the higher the blood pressure and insulin level. Thus, the higher cardiovascular rates seen with higher insulin levels may be caused by an increase in other risk factors such as hypertension, rather than insulin itself.

Such a relationship is often ignored in articles that claim an association between insulin resistance and cardiovascular events. For example, in the Veteran Affairs-HDL Intervention Trial (VA-HIT), the authors concluded that "the occurrence of a new cardiovascular event was dependent on ... the presence or absence of insulin resistance." is interesting to note that the group with insulin resistance had an average body mass index of over 31 while those without insulin resistance had an average body mass index of only $27 .{ }^{10}$ Furthermore, there was no reporting of blood pressure values. Although it is possible that higher levels of insulin increased the rate of cardiovascular events in this population, it is equally plausible than other factors such as hypertension were responsible.

This then begs the question of whether hypertension is a byproduct of hyperinsulinemia. Although no unequivocal data to answer this question exist, multiple experiments in dogs have shown this not to be the case. There were no pressor effects noted in normal dogs infused with insulin, or in dogs with a $70 \%$ reduction in kidney mass on a high salt diet. ${ }^{11}$ Interestingly, chronic hyperinsulinemia actually caused a reduction in total peripheral vascular resistance as well as arterial pressure. ${ }^{11}$ This decrease in vascular resistance disappeared when the dogs were made obese via a high-fat diet. ${ }^{11}$ This series of experiments suggests that in dogs, hyperinsulinemia does not cause hypertension. Whether or not this translates into humans remains to be seen. However, a cross-sectional relational study found obesity to be an independent risk factor for left ventricular hypertrophy, but not insulin resistance or fasting insulin levels. ${ }^{12}$ These data seem to suggest that obesity and its complications are responsible for cardiovascular risk, not merely high insulin levels.

Although there is a theoretical concern of a mitogenic effect of insulin analogs, this concern appears limited to the long-acting insulin glargine. ${ }^{13}$ Glargine appears to be a more potent stimulus of DNA synthesis in human osteosarcoma cell lines than the native insulin molecule; the rapid-acting analogs appear to be equivalent to regular insulin. ${ }^{14}$ Some have postulated that mitogenic potency is related to the halflife of the receptor-ligand binding complex, which would explain why the rapid-acting analogs do not appear to have as much theoretical mitogenic effect as the long-acting analog glargine. ${ }^{15}$

\section{Does intensive diabetes therapy with insulin improve vascular events?}

Recently, there have been several large prospective studies examining the relationship between the treatment of hyperglycemia and vascular complications. The first major study to associate a decrease in vascular events with glycemic control was the United Kingdom Prospective Diabetes Study (UKPDS). This prospective observational study included 4585 type 2 diabetic subjects. It concluded that each $1 \%$ reduction in mean $\mathrm{HbA}_{1 \mathrm{c}}$ was associated with a risk reduction of $21 \%$ for deaths related to diabetes, $14 \%$ for myocardial infarction, and $37 \%$ of microvascular complications. ${ }^{16}$

These patients were followed for another 10 years without diabetic treatment manipulation by the researchers. Despite the difference in $\mathrm{HbA}_{1 \mathrm{c}}$ disappearing after one year, the group intensively treated initially with insulin or sulfonylurea still had a $24 \%$ risk reduction for microvascular disease, a 15\% in risk reduction for myocardial infarction, and a 13\% reduction for death from any cause. ${ }^{17}$ Because patients initially treated with metformin also had risk reductions, the authors of this study concluded that this legacy effect was not the result of insulin, but rather a possible reduction in advanced glycation end products from the initial intensive treatment of hyperglycemia. ${ }^{18}$

The Diabetes Control and Complications Trial (DCCT) and the subsequent Epidemiology of Diabetes Interventions and Complications (EDIC) illustrated that intensive insulin therapy in type 1 diabetic patients reduced the major diabetic complications of neuropathy, nephropathy, and retinopathy. ${ }^{19}$ Intensive insulin therapy was associated with less cardiovascular disease as evidenced by decreased intima-media thickness and lower coronary artery calcium accumulation. ${ }^{20}$ Intensive insulin treatment also decreased the risk of any cardiovascular disease by $42 \%$ and the risk of non-fatal stroke, myocardial infarction, or cardiovascular death by $57 \%$ in type 1 diabetic subjects. ${ }^{21}$

Intensive insulin treatment was also shown to be beneficial to those with the worst vascular disease. Diabetic patients who suffered an acute myocardial infarction had an absolute reduction in mortality of $11 \%$ when treated with intensive insulin therapy. ${ }^{22}$ 
Other studies have been less convincing. The recent Action in Diabetes and Vascular Disease (ADVANCE) trial was aimed to address the effect of intensive glucose control on vascular outcomes in subjects with type 2 diabetes. The researchers randomly assigned 11,140 type 2 diabetic patients to standard glucose control or intensive glucose control using to a $\mathrm{HbA}_{1 \mathrm{c}}$ of $6.5 \%$ or less. ${ }^{23}$ Although the first line drug was a sulfonylurea, $40.5 \%$ of subjects in the intensive arm and $24.1 \%$ of subjects in the standard arm ended up on insulin. ${ }^{23}$ After a median of 5 years of followup, the researchers found that intensive glucose control led to a reduction in nephropathy (4.1 versus $5.2 \%$ ), but had no effect on retinopathy, major macrovascular events, or death from cardiovascular causes. ${ }^{23}$

The Action to Control Cardiovascular Risk in Diabetes (ACCORD) trial also attempted to address the relationship between vascular events and glucose control in patients with type 2 diabetes. One distinguishing feature of this trial is that all subjects had to have either established cardiovascular disease or additional cardiovascular risk factors. Therefore, it was aimed for secondary instead of primary prevention of vascular disease. 10,251 subjects were randomly assigned to intensive therapy $\left(\mathrm{HbA}_{1 \mathrm{c}}<6 \%\right)$ or standard therapy $\left(\mathrm{HbA}_{1 \mathrm{c}} 7.0 \%-7.9 \%\right) .{ }^{24}$ This portion of the study (a blood pressure arm is still ongoing) was discontinued after a mean of 3.5 years of follow up due to an increase in total mortality (257) in the intensive group as opposed to the standard group (203). ${ }^{24}$ Interestingly, the number of events in the primary outcome (non-fatal myocardial infarction, non-fatal stroke, or death from cardiovascular cause) was actually lower in the intensive group (352) as compared to the standard group (371). ${ }^{24}$ This calls into debate whether the increase in deaths was due to vascular complications, or if the increase would have panned out had the trial not ceased prematurely. Thus far, there has been no concrete explanation for the increase in mortality seen in the intensive arm. However, some postulate that hypoglycemia may have contributed to the cardiovascular events. ${ }^{25}$ If so, then theoretically, in comparison to regular insulin, the lower hypoglycemic rate associated with the rapid-acting analogs could provide a cardiovascular benefit. ${ }^{26}$

A third trial, the Veterans Affairs Diabetes Trial, also examined the effects of intensive blood glucose control on cardiovascular events. The intensive goal in this trial was defined by a goal $\mathrm{HbA}_{1 \mathrm{c}}$ of $<7.5 \%$ and a standard arm goal of $\mathrm{HbA}_{1 \mathrm{c}}<8.3 \%$, while controlling lipids and blood pressure in both arms. ${ }^{27}$ This trial was conducted at 20 different VA centers with 1791 patients with a mean follow-up of
6.25 years. In this study, there was no significant reduction in cardiovascular events in the intensive arm. ${ }^{28}$

Thus, the effect of aggressive control of blood glucose on cardiovascular events remains debatable. UKPDS and DCCT/EDIC have shown that tight control in younger patients in recently diagnosed disease was beneficial, whereas ADVANCE, ACCORD, and VADT have shown that in older patients with long-standing disease the benefits of tight glucose control are not as convincing. Diabetes control may very well have different effects on different cohorts.

\section{How might insulin reduce vascular events?}

Insulin's beneficial effects on the vascular system are likely due to actions on the endothelium (see Table 1). Insulin has been shown to be a potent vasodilator by causing an increased production of nitric oxide. ${ }^{29}$ Insulin induces the expression of nitric oxide synthetase, which converts arginine to nitric oxide. ${ }^{30}$ Separate experiments then illustrated that nitric oxide levels increase in a dose dependent manner in human vein endothelial cells as well as human aortic endothelial cells. ${ }^{31,32}$ These observations were translated in humans when researchers infused insulin into normal subjects' arteries, and found that blood flow increases in a dose dependent manner with insulin. ${ }^{29}$

However, in type 2 diabetic as well as non-diabetic obese patients, the vasodilatory effect of insulin is blunted..$^{33,34}$ Whether this decrease in vasodilation is due to insulin resistance or if insulin resistance is a byproduct of a decrease in skeletal muscle perfusion and insulin-mediated glucose uptake remains to be seen.

In addition to effects on vascular tone, diabetes also has an inflammatory component, characterized by endothelial dysfunction and elevated adhesion molecules. ${ }^{35}$ Insulin helps to counteract this inflammation, by suppressing the expression of adhesion molecules, allowing monocytes to couple with endothelial cells, releasing monocyte chemoattractant protein-1 (MCP-1). This in turn attracts more monocytes which then intermingle with low-density lipoprotein cholesterol, forming foam cells. ${ }^{29}$ Insulin also reverses the increase in the adhesion molecule e-selectin seen in type 2 diabetic subjects. ${ }^{36}$

Along with suppressing MCP-1 and e-selectin, insulin also suppresses nuclear factor kappa B (NF-kB) ${ }^{37} \mathrm{NF}-\mathrm{kB}$ is a central figure in inflammation, responsible for the production of cytokines, enzymes, and adhesion molecules. ${ }^{38}$ When human aortic endothelial cells were incubated with insulin, intranuclear NF-kB binding activity and MCP-1 
Table I Comparison of rapid-acting insulin analogs with regular insulin

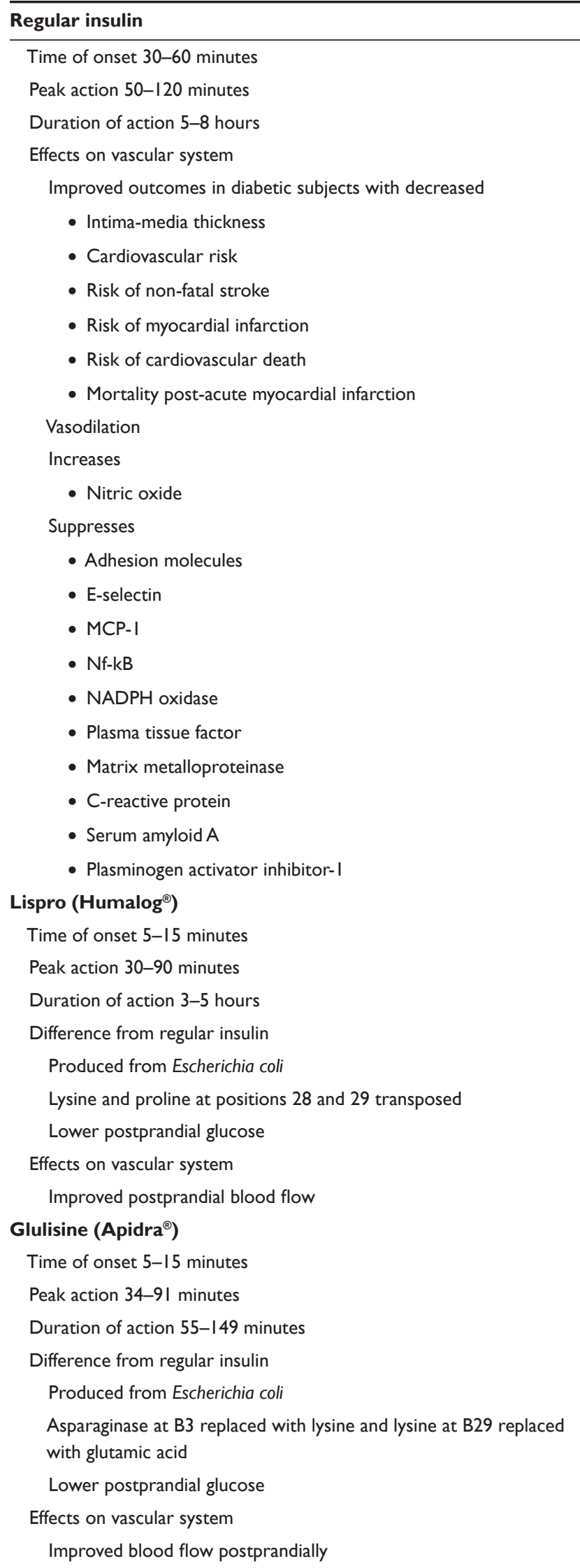

(Continued)
Table I (Continued)

\begin{tabular}{l}
\hline Aspart $\left(\mathbf{N o v o l o g}^{\circledR}\right)$ \\
\hline Time of onset 5-15 minutes \\
Peak action $40-50$ minutes \\
Duration of action 3-5 hours \\
Difference from regular insulin \\
Produced from Saccharomyces cervisiae \\
Proline replaced with aspartic acid at B28 \\
Lower postprandial glucose \\
Effects on vascular system \\
No changes in fasting lipid profile, apolipoproteins, fibrinogen, \\
Plasminogen activator inhibitor-I, E-selectin, or homocysteine when \\
compared to regular insulin.
\end{tabular}

Note: Time values assume subcutaneous bolus injection.

mRNA expression were both suppressed. ${ }^{37}$ This decrease in MCP-1 was replicated in vivo in obese subjects, in whom the translocation of NF-kB was inhibited by insulin infusion. ${ }^{39}$ Insulin also decreases NADPH oxidase, plasma tissue factor, and matrix metalloproteinase. ${ }^{29}$

Insulin has been shown to decrease the inflammatory marker C-reactive protein in critically ill patients. ${ }^{40} \mathrm{In}$ patients suffering from an acute myocardial infarction, insulin has antiinflammatory and pro-fibrinolytic effects as evidenced by a blunting of the increase in high-sensitivity $\mathrm{C}$-reactive protein, serum amyloid A, and plasminogen activator inhibitor-1..$^{41}$

\section{Are the effects of insulin a class effect?}

It is unclear if the effects of insulin are unique to a certain formulation or are class effects that extend to the rapidacting insulin analogs. Table 1 summarizes the experimental evidence of the various formulations of short-acting insulin. One type of rapid-acting insulin is lispro (Humalog ${ }^{\circledR}$, Eli Lilly and Company). Lispro is a human insulin analog manufactured from Escherichia coli with the amino acids at positions 28 and 29 reversed. ${ }^{42}$ This reversal of lysine and proline allows the insulin to be absorbed much faster than regular insulin from subcutaneous tissue. Lispro has a peak action of 30 to 90 minutes. ${ }^{42}$ Because of its fast onset, lispro is often used as a bolus insulin to address postprandial glucose excursions. Like the other rapid-acting analogs, it can be used alone in an insulin pump, or in combination with a longer acting insulin formulation. It is marketed by itself as well as pre-mixed with lispro protamine, a crystallized form of lispro made by combining lispro with protamine sulfate, lengthening its duration of action. ${ }^{43}$ Clinical guidelines and algorithms for the use of rapid-acting analogs in diabetic patients are beyond the scope of this article. 
There are some data to show that lispro is beneficial to the vascular system. Specifically, lispro improves microvascular blood flow in postprandial type 1 diabetic patients. ${ }^{44}$ In this study, 20 non-diabetic patients and 20 diabetic patients had their skin microvascular blood flow measured by laser Doppler flux every 30 minutes after a standardized test meal. The researchers found that microvascular blood flow was impaired in type 1 diabetic patients given regular insulin, but when given insulin lispro, the microvascular blood flow improved to mimic the non-diabetic subject. This is thought to be due to the treatment of acute hyperglycemia, which stimulates the production of free radicals and augments thrombin formation. ${ }^{44}$ Hyperglycemia is also thought to create an increase in adhesion molecules and endothelin, while decreasing levels of nitric oxide. ${ }^{45}$ Because lispro has a faster onset of action than regular insulin, it reduces these postprandial excursions better, and is more likely to counteract the increase in adhesion molecules and decrease in nitric oxide. ${ }^{46}$

Similar to lispro, glulisine (Apidra ${ }^{\circledR}$, Sanofi-Aventis) has also been shown to have beneficial effects on surrogate vascular markers. Glulisine is a rapid-acting insulin homologous with regular human insulin save for the amino acid asparagine at position B3 replaced with lysine and the lysine at position B29 substituted with glutamic acid. ${ }^{47}$ This analog is produced by recombinant DNA using Escherichia coli, and it is employed to lower blood glucose in a similar fashion as lispro. In a microvascular blood flow study similar to the one described above using lispro and regular insulin, glulisine was compared to regular insulin in 15 type 2 diabetic subjects after a liquid meal challenge. Serial laser Doppler fluxometry was then performed. Like the results in the lispro study, glulisine-reated subjects had higher postrandial insulin levels, lower glucose excursions, and higher microvascular blood flow. ${ }^{48}$ Again, without any head to head comparison studies, it is impossible to say that the short-acting insulin analogs are equal. Yet, the fact that both glulisine and lispro had similar effects in a similarly designed study suggests a class effect benefit from treating postprandial glucose excursions.

Although a similar study using laser fluxometry has not been published using aspart (NovoLog ${ }^{\circledR}$, Novo Nordisk Inc.), there have been other studies examining the effect of aspart on vascular risk factors. Aspart is similar in composition to regular human insulin, save for the substitution of the amino acid proline with aspartic acid in position $\mathrm{B} 28{ }^{49} \mathrm{It}$ is produced using recombinant DNA technology using Saccharomyces cerevisiae (baker's yeast) and is used to treat hyperglycemia in a similar fashion as lispro and glulisine. Despite the similar function and onset of action, the data on aspart's effects on the vascular system are not as clear as with the other rapid-acting analogs. Twenty-one patients with insulin-treated type 2 diabetes were given neutral protamine Hagedorn (NPH) insulin and pre-prandial regular insulin or aspart for 6 weeks. ${ }^{50}$ At the end of the study, although there was no statistical difference in hemoglobin $\mathrm{A}_{1 \mathrm{c}}\left(\mathrm{HbA}_{1 \mathrm{c}}\right)$, the aspart group had a significantly lower postprandial (90 minutes after a meal) average glucose $(7.9 \mathrm{mmol} / \mathrm{L}$ versus $9.3 \mathrm{mmol} / \mathrm{L}) .{ }^{50}$ Despite this decrease in glucose excursions, there were no statistical differences in markers of vascular risk (fasting lipid profile, apolipoproteins, fibrinogen, plasminogen activator inhibitor-1, E-selectin, or homocysteine). However this study has several limitations to preclude the conclusion that treating postprandial glucose excursions has no effect on vascular risk. This study's conclusion may reflect a lack of power, brief study duration, or even a difference between the effects of distinct insulin analogs on vascular risk.

\section{Does treatment of postprandial glucose help?}

The rapid-acting insulins' superiority lies in their ability to treat postprandial glucose and excursions. Is such an ability clinically relevant? Fortunately, several studies highlight how postprandial excursions and high glucose variability affect the vascular system. One such study involved type 1 diabetic patients who underwent myocardial perfusion studies while either on a euglycemic or hyperglycemic hyperinsulinemic clamp. The myocardial perfusion reserve was significantly decreased when subjects were hyperglycemic. ${ }^{51}$

Just as chronic diabetes is associated with inflammation, acute hyperglycemia has also been shown to cause an increase in proinflammatory cytokines such as tumor necrosis factor-alpha, interleukin (IL)-6, IL-1 beta, and IL-8, which leads to vascular inflammation. ${ }^{52}$ This increase in cytokines was counteracted by insulin infusion and a return to normoglycemia. ${ }^{52}$

By mitigating hyperglycemia with a faster onset of action than regular insulin, some have argued that rapid-acting insulin may have favorable cardiovascular effects. ${ }^{53,54}$ One argument is that using premixed lispro combinations lower postprandial glucose better than regular insulin combinations, and therefore may reduce CVD risk. ${ }^{54}$ When lispro was added to bedtime NPH insulin and compared to twice-daily $\mathrm{NPH}$ in type 2 diabetic subjects, the lispro-treated group experienced lower postprandial glucose, lower $\mathrm{HbA}_{1 \mathrm{c}}$, lower triglycerides, low-density lipoprotein cholesterol (LDL), and higher high-density lipoprotein cholesterol (HDL). ${ }^{53}$ The authors surmised that this favorable milieu might have 
a positive cardiovascular effect. In addition, a recent study was performed comparing the long-acting insulin detemir (Levemir $^{\circledR}$, Novo Nordisk Inc.) to the rapid-acting aspart. It found that patients using a prandial bolus of insulin aspart had a lower $\mathrm{HbA}_{1 \mathrm{c}}$ than those treated with basal detemir. ${ }^{55}$ These two studies would suggest that the postprandial excursions can drive overall blood glucose control, and that by treating this variability, vascular events could be mitigated. Reducing glycemic variability could also lessen glycemic events due to fewer episodes of hypoglycemia. A recent study of 100 type 1 diabetic patients followed for 11 years found a strong correlation between glycemic variability and hypoglycemic unawares. ${ }^{56}$ It is possible that using insulin analogs could reduce glycemic variability and hypoglycemic episodes, whereby overall glucose control and vascular events are improved. However, this theory can only be answered with a prospective long-term study with an extremely large sample size using rapid-acting insulin.

On the other hand, specifically targeting postprandial glucose may not have a clinically significant vascular effect. The hyperglycemia and its effect after acute myocardial infarction on cardiovascular outcomes in patients with type 2 diabetes mellitus (HEART2D) attempted to randomize 1355 subjects with type 2 diabetes and acute myocardial infarction to either pre-meal insulin lispro with basal NPH insulin, (with a target 2-hour postprandial blood glucose of $<135 \mathrm{mg} / \mathrm{dL}$ ) or basal insulin only (NPH insulin twice daily, insulin glargine once daily, or pre-mixed human insulin [70\% NPH/30\% regular] twice daily) targeting fasting. ${ }^{57}$ Although both groups ended up with similar $\mathrm{HbA}_{1 \mathrm{c}}$ values (7.6\%), and the lispro-treated group had statistically fewer postprandial excursions, there was no significant difference in cardiovascular events between the two groups and the study was stopped for "futility." 58 These data would suggest that glycemic variability does not increase cardiovascular events, although the sample size of the study was relatively small and it experienced a high drop out rate.

\section{Conclusion}

In summary, the UKPDS has shown that treating diabetes to a goal $\mathrm{HbA}_{1 \mathrm{c}}<7 \%$ will decrease some vascular events. Intensively treating diabetes to $<6 \%$ is debatable and difficult given present treatment modalities. Individualized goals should be designed between physician and patient. In meeting these goals, insulin has been shown to be beneficial by not only by treating hyperglycemia, but also by providing vasodilatory and anti-inflammatory effects. Although not proven, these effects likely extend to the rapid-acting analogs. With its faster onset of action and lower postprandial glucose excursions, rapid-acting insulin appears superior to regular insulin, and could possibly reduce vascular events further.

\section{Disclosures}

The authors declare no conflicts of interest.

\section{References}

1. Clearinghouse NDI. National Diabetes Statistics 2007. www.diabetes. niddk.nih.gov. Accessed September 8, 2008.

2. Nathan DM. Clinical practice. Initial management of glycemia in type 2 diabetes mellitus. N Engl J Med. 2002;347(17):1342-1349.

3. Shear K. Weight-loss more effective than intensive insulin therapy for type 2 diabetics, according to expert. UT Southwestern News [March 11, 2008; http://www.utsouthwestern.edu/utsw/cda/dept353744/ files/449942.html. Accessed September 8, 2008.

4. Unger RH. Reinventing type 2 diabetes: pathogenesis, treatment, and prevention. JAMA. 2008;299(10):1185-1187.

5. Pyorala M, Miettinen H, Laakso M, Pyorala K. Plasma insulin and all-cause, cardiovascular, and noncardiovascular mortality: the 22-year follow-up results of the Helsinki Policemen Study. Diabetes Care. 2000;23(8):1097-1102.

6. Ducimetiere P, Eschwege E, Papoz L, Richard JL, Claude JR, Rosselin G. Relationship of plasma insulin levels to the incidence of myocardial infarction and coronary heart disease mortality in a middleaged population. Diabetologia. 1980;19(3):205-210.

7. Leonetti F, Iozzo P, Giaccari A, et al. Absence of clinically overt atherosclerotic vascular disease and adverse changes in cardiovascular risk factors in 70 patients with insulinoma. $J$ Endocrinol Invest. 1993;16(11):875-880.

8. Semenkovich CF. Insulin resistance and atherosclerosis. J Clin Invest. 2006;116(7):1813-1822.

9. Aristizabal D, Gallo J, Fernandez R, Restrepo MA, Zapata N, Correa M. The insulin gradient phenomenon: a manifestation of the effects of body weight on blood pressure and insulin resistance. J Cardiometab Syndr. 2008;3(4):218-223.

10. Robins SJ, Rubins HB, Faas FH, et al. Insulin resistance and cardiovascular events with low HDL cholesterol: the Veterans Affairs HDL Intervention Trial (VA-HIT). Diabetes Care. 2003;26(5):1513-1517.

11. Brands MW, Hall JE. Insulin resistance, hyperinsulinemia, and obesityassociated hypertension. J Am Soc Nephrol. 1992;3(5):1064-1077.

12. Ebinc H, Ebinc FA, Ozkurt ZN, Dogru T, Yilmaz M. Relationship of left ventricular mass to insulin sensitivity and body mass index in healthy individuals. Acta Cardiol. 2006;61(4):398-405.

13. Miles HL, Acerini CL. Insulin analog preparations and their use in children and adolescents with type 1 diabetes mellitus. Paediatr Drugs. 2008;10(3):163-176.

14. Kurtzhals P, Schaffer L, Sorensen A, et al. Correlations of receptor binding and metabolic and mitogenic potencies of insulin analogs designed for clinical use. Diabetes. 2000;49(6):999-1005.

15. Hansen BF, Danielsen GM, Drejer K, et al. Sustained signalling from the insulin receptor after stimulation with insulin analogues exhibiting increased mitogenic potency. Biochem J. 1996;315(1):271-279.

16. Stratton IM, Adler AI, Neil HA, et al. Association of glycaemia with macrovascular and microvascular complications of type 2 diabetes (UKPDS 35): prospective observational study. BMJ. 2000;321(7258):405-412.

17. Holman RR, Paul SK, Bethel MA, Matthews DR, Neil HAW. 10-year follow-up of intensive glucose control in type 2 diabetes. N Engl J Med. 2008;359(15):1577-1589.

18. Goh S-Y, Cooper ME. The role of advanced glycation end products in progression and complications of diabetes. J Clin Endocrinol Metab. 2008;93(4):1143-1152. 
19. Ginsberg BJ, Mazze R. Clinical consequences of the Diabetes Control and Complications Trial. N J Med. 1994;91(4):221-224.

20. Nathan DM, Lachin J, Cleary P, et al. Intensive diabetes therapy and carotid intima-media thickness in type 1 diabetes mellitus. $N$ Engl $J$ Med. 2003;348(23):2294-2303.

21. Nathan DM, Cleary PA, Backlund JY, et al. Intensive diabetes treatment and cardiovascular disease in patients with type 1 diabetes. $N$ Engl $J$ Med. 2005;353(25):2643-2653.

22. Malmberg K. Prospective randomised study of intensive insulin treatment on long term survival after acute myocardial infarction in patients with diabetes mellitus. DIGAMI (Diabetes Mellitus, Insulin Glucose Infusion in Acute Myocardial Infarction) Study Group. BMJ. 1997;314(7093):1512-1515.

23. ADVANCE Collaborative Group; Patel A, MacMahon S, Chalmers J, et al. Intensive blood glucose control and vascular outcomes in patients with type 2 diabetes. N Engl J Med. 2008;358(24):2560-2572.

24. Action to Control Cardiovascular Risk in Diabetes Study Group, Gerstein HC, Miller ME, Byington RP, et al. Effects of intensive glucose lowering in type 2 diabetes. $N$ Engl J Med. 2008;358(24):2545-2559.

25. Dluhy RG, McMahon GT. Intensive glycemic control in the ACCORD and ADVANCE trials. $N$ Engl J Med. 2008;358(24):2630-2633.

26. Rossetti P, Porcellati F, Fanelli CG, Perriello G, Torlone E, Bolli GB. Superiority of insulin analogues versus human insulin in the treatment of diabetes mellitus. Arch Physiol Biochem. 2008;114(1):3-10.

27. Abraira C, Duckworth WC, Moritz T. Glycaemic separation and risk factor control in the Veterans Affairs Diabetes Trial: an interim report. Diabetes Obes Metab. 2009;11(2):150-156.

28. Ismail-Beigi F, Moghissi ES. Glycemia management and cardiovascular risk in type 2 diabetes: an evolving perspective. Endocr Pract. 2008;14(5):639-643.

29. Fonseca VA. The effects of insulin on the endothelium. Endocrinol Metab Clin North Am. 2007;36S2:20-26.

30. Dandona P, Aljada A, Mohanty P. The anti-inflammatory and potential anti-atherogenic effect of insulin: a new paradigm. Diabetologia. 2002;45(6):924-930.

31. Aljada A, Dandona P. Effect of insulin on human aortic endothelial nitric oxide synthase. Metabolism. 2000;49(2):147-150.

32. Zeng G, Quon MJ. Insulin-stimulated production of nitric oxide is inhibited by wortmannin. Direct measurement in vascular endothelial cells. J Clin Invest. 1996;98(4):894-898.

33. Baron AD. Insulin and the vasculature - old actors, new roles. J Investig Med. 1996;44(8):406-412.

34. Baron AD. Hemodynamic actions of insulin. Am J Physiol. 1994; 267(2 Pt 1):E187-202.

35. Libby P, Ridker PM, Maseri A. Inflammation and atherosclerosis. Circulation. 2002;105(9):1135-1143.

36. Albertini JP, Valensi P, Lormeau B, et al. Elevated concentrations of soluble E-selectin and vascular cell adhesion molecule-1 in NIDDM. Effect of intensive insulin treatment. Diabetes Care. 1998;21(6):1008-1013.

37. Aljada A, Ghanim H, Saadeh R, Dandona P. Insulin inhibits NFkappaB and MCP-1 expression in human aortic endothelial cells. $J$ Clin Endocrinol Metab. 2001;86(1):450-453.

38. Barnes PJ, Karin M. Nuclear factor-kappaB: a pivotal transcription factor in chronic inflammatory diseases. N Engl J Med. 1997;336(15): 1066-1071.

39. Dandona $\mathrm{P}, \mathrm{Aljada} \mathrm{A}$, Mohanty $\mathrm{P}$, et al. Insulin inhibits intranuclear nuclear factor kappaB and stimulates IkappaB in mononuclear cells in obese subjects: evidence for an anti-inflammatory effect? $J$ Clin Endocrinol Metab. 2001;86(7):3257-3265.

40. Hansen TK, Thiel S, Wouters PJ, Christiansen JS, Van den Berghe G. Intensive insulin therapy exerts antiinflammatory effects in critically ill patients and counteracts the adverse effect of low mannose-binding lectin levels. J Clin Endocrinol Metab. 2003;88(3):1082-1088.
41. Chaudhuri A, Janicke D, Wilson MF, et al. Anti-inflammatory and profibrinolytic effect of insulin in acute ST-segment-elevation myocardial infarction. Circulation. 2004;109(7):849-854.

42. Humalog Package Insert. http://pi.lilly.com/us/humalog-pen-pi.pdf. Accessed September 8, 2008.

43. Humalog 75/25. Package Insert. http://pi.lilly.com/us/humalog7525pi.pdf. Accessed September 8, 2008.

44. Forst T, Forst S, Strunk K, et al. Impact of insulin on microvascular blood flow and endothelial cell function in the postprandial state in patients with Type 1 diabetes. $J$ Diabetes Complications. 2005;19(3):128-132.

45. Lefebvre PJ, Scheen AJ. The postprandial state and risk of cardiovascular disease. Diabet Med. 1998;(15 Suppl)4:S63-S68.

46. Gale EA. A randomized, controlled trial comparing insulin lispro with human soluble insulin in patients with Type 1 diabetes on intensified insulin therapy. The UK Trial Group. Diabet Med. 2000;17(3):209-214.

47. Apidra Prescribing Information. http://products.sanofi-aventis.us/ apidra/apidra.html. Accessed September 29, 2008.

48. Hohberg C, Forst T, Larbig M, et al. Effect of Insulin Glulisine on Microvascular Blood Flow and Endothelial Function in the Postprandial State. Diabetes Care. 2008;31(5):1021-1025.

49. Novolog Prescribing Information. Prescribing Information. Available at: http://www.novolog.com/NovoLog_Prescribing_Info.pdf. Accessed September 29, 2008

50. Gallagher A, Home PD. The effect of improved post-prandial blood glucose control on post-prandial metabolism and markers of vascular risk in people with Type 2 diabetes. Diabetes Res Clin Pract. 2005;67(3):196-203.

51. Srinivasan M, Herrero P, McGill JB, et al. The effects of plasma insulin and glucose on myocardial blood flow in patients with type 1 diabetes mellitus. J Am Coll Cardiol. 2005;46(1):42-48.

52. Stentz FB, Umpierrez GE, Cuervo R, Kitabchi AE. Proinflammatory cytokines, markers of cardiovascular risks, oxidative stress, and lipid peroxidation in patients with hyperglycemic crises. Diabetes. 2004;53(8):2079-2086.

53. Ceriello A, Del Prato S, Bue-Valleskey J, et al. Premeal insulin lispro plus bedtime NPH or twice-daily NPH in patients with type 2 diabetes: acute postprandial and chronic effects on glycemic control and cardiovascular risk factors. J Diabetes Complications. 2007;21(1):20-27.

54. Giugliano D, Ceriello A, Razzoli E, Esposito K. Defining the role of insulin lispro in the management of postprandial hyperglycaemia in patients with type 2 diabetes mellitus. Clin Drug Investig. 2008;28(4):199-210.

55. Holman RR, Thorne KI, Farmer AJ, et al. Addition of biphasic, prandial, or basal insulin to oral therapy in type 2 diabetes. $N$ Engl $J$ Med. 2007;357(17):1716-1730.

56. Bragd J, Adamson U, Backlund LB, Lins PE, Moberg E, Oskarsson P. Can glycaemic variability, as calculated from blood glucose selfmonitoring, predict the development of complications in type 1 diabetes over a decade? Diabetes Metab. 2008;34(6):612-616.

57. Milicevic Z, Raz I, Strojek K, et al. Hyperglycemia and its effect after acute myocardial infarction on cardiovascular outcomes in patients with Type 2 diabetes mellitus (HEART2D) Study design. $J$ Diabetes Complications. 2005;19(2):80-87.

58. Hyperglycemia and Its Effect After Acute Myocardial Infarction on Cardiovascular Outcomes in Patients With Type 2 Diabetes (HEART2D) study. American Diabetes Association 68th Scientific Sessions: Late Breaking Clinical Studies. Presented June 9, 2008. 
